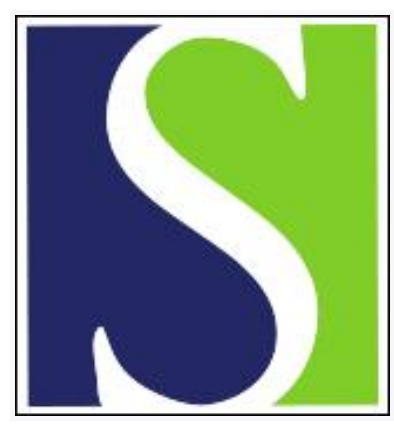

Scand J Work Environ Health 1981;7(4):271-276

https://doi.org/10.5271/sjweh.2547

Issue date: Dec 1981

Accumulation of fibers in the eyes of workers handling man-made mineral fiber products.

by Schneider T, Stokholm J

Key terms: accumulation; biological monitoring; conjunctival mucus; eye; fiber; irritant; man-made mineral fiber; man-made mineral fiber product; mineral fiber; passive dust sampler; skin; worker

This article in PubMed: www.ncbi.nlm.nih.gov/pubmed/7347912

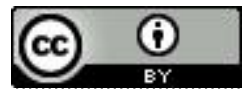




\title{
Accumulation of fibers in the eyes of workers handling man-made mineral fiber products
}

\author{
by Thomas Schneider, MSc, Jens Stokholm, MD ${ }^{1}$
}

\begin{abstract}
SCHNEIDER T, STOKHOLM J. Accumulation of fibers in the eyes of workers handling man-made mineral fiber products. Scand $j$ work environ health 7 (1981) $271-276$. The external eye can be considered a passive dust sampler. Foreign material deposited in the conjunctival cul-de-sac becomes enclosed by mucous fibrils. The fibrils adhere to the conjunctival mucous thread. Hence, by removing the mucus, one can study, eg, man-made mineral fibers deposited in the eye. In this study a simple method for this purpose has been developed, and the sampling characteristics of the eye for nonrespirable fibers is described. The numbers of nonrespirable fibers accumulated in the eyes correlated with the total dust exposure dose (correlation coefficient $r=0.92$ ) and with the nonrespirable fiber exposure dose $(r=0.82)$. There was no correlation between the airborne fiber concentration and the number of fibers in the mucous thread alone.
\end{abstract}

Key terms: biological monitoring, conjunctival mucus, irritants, passive dust sampler, skin.

The mucosa of the airways and of the external eye are primary target organs for air pollutants. Since routine ophthalmologic examinations, including slit-lamp microscopy (7), can be used under field conditions, it would be an advantage to be able to investigate air pollution effects by studying the eyes. The external eye can be considered a passive dust sampler. If the dust sampled by the eye can be determined quantitatively, dose-effect relationships can be established.

The purpose of this study was to develop a simple method for the determination of the amount of man-made mineral fibers accumulated in the human eye at the workplace. The method is based on sampling the mucus from the eye.

\section{Previous studies on the deposition of dust and fibers in the eyes}

The surface of the eye is covered by a very thin translucent layer of mucus.

1 National Institute of Occupational Health, Hellerup, Denmark.

Reprint requests to: $\mathrm{Mr} \mathrm{T}$ Schneider, National Institute of Occupational Health, Baunegårdsvej 73, DK-2900 Hellerup, Denmark.
Foreign bodies deposited on the cornea or the conjunctiva are rapidly enclosed in mucous fibrils during blinking. Blinking gradually causes these fibrils to adhere to the mucous thread, which is always present in the lower conjunctival fornix of normal eyes. During blinking, the mucous thread moves towards the inner corner of the eye and further on to the skin, where it dries to become "sleepy seeds." The mucous thread thus acts as a conveyor belt for foreign bodies, which are removed from the eye and left on the skin in the inner corner of the eye (5).

Gibson \& Vincent (2) have studied the impaction of dust in the eyes of human subjects, especially of fly dust in coal mines. They showed that, for a given set of conditions, there is an upper limit for the size of particles entering the eyes of experimental subjects, and this limit is considerably less than for particles entering the eyes of an inert unblinking dummy. They also found that the size distribution of the dust particles entering the eyes of a dummy was less than for the airborne dust sampled isokinetically. The size distribution of the particles deposited in the eye was determined from samples of 
dust taken from the inner corner of the eyes with sterile cotton buds.

Thick and long fibers become airborne (9) during the application of man-made mineral fiber insulation products. Irritation in the upper airways from these fibers is well known, and it could be expected that particles with such particular shapes could cause irritation of the eyes as well. Lucas (4) reviewed ocular effects and concluded that the ocular hazards of airborne fibrous glass dust, if they occur, are remarkably rare and, under ordinary conditions of exposure, do not pose a problem. However, no detailed ophthalmologic investigation of the effect of fibers on the cornea or conjunctiva have been reported, and no reports exist on the size and total number of fibers which can accumulate in the eyes during exposure to fibrous dust.

Nearly $100 \%$ of the man-made mineral fibers with diameters exceeding $3 \mu \mathrm{m}$ are deposited in the nasopharyngeal region after entering the nose (3). The potential for skin irritation depends on fiber diameter as follows: less than $5 \mu \mathrm{m}$, slight, if any; 5 to $7.5 \mu \mathrm{m}$, moderate; 7.5 to $10 \mu \mathrm{m}$, high (8). The irritation known to occur in the upper airways and on the skin is thus linked to fibers with diameters exceeding $3 \mu \mathrm{m}$ (nonrespirable fibers). Therefore we decided to focus on the deposition of nonrespirable fibers.

\section{Materials and methods}

Two work situations, in which none of the workers wore glasses or contact lenses, were studied. The first included the secondary processes of the manufacturing of man-made mineral fiber insulation (cutting, packing, storing, granulating). The manufacturing conditions were constant from Monday through Thursday. On Thursday dust measurements were made during the whole 8-h shift. The mucus was sampled immediately after the end of the exposure period of that shift.

The second was a standardized task, in which two persons at a time insulated the walls and ceiling of an unventilated chamber [dimensions $2.8 \times 2.9 \times 2.8$ (height) $\mathrm{m}$ ]. Two persons used conventional insulating material (material $A$ ) for a period of $2 \mathrm{~h}$; then two others used material for fire proofing (material B) for a period of $2 \mathrm{~h}$. Consecutive dust samples were taken during periods ranging from 21 to $45 \mathrm{~min}$. The mucus was sampled after exposure periods ranging from 35 to 70 $\min$.

\section{Evaluation of airborne dust}

Total dust measurements were made with personal samplers using Sartorius ${ }^{\circledR} 11401$ membrane filters in Millipore ${ }^{\circledR}$ field monitors with lids; the samplers were fixed to the lapels of the workers. The flow rate was $1.9 \mathrm{l} / \mathrm{min}$, and the hole was pointing forward. The diameter was enlarged to $5.7 \mathrm{~mm}$ in order to give the required air velocity of $1.25 \mathrm{~m} / \mathrm{s}( \pm 10 \%)$ in the inlet. The filters were weighed in a room with a constant temperature $\left(25^{\circ} \mathrm{C}\right)$ and relative humidity $(50 \%)$ for the determination of total mass. The filters were then ashed in a low-temperature plasma asher, and the fibers were redispersed in trichloroethylene by gentle shaking and filtered onto a Sartorius ${ }^{\circledR} 11406$ membrane filter for fiber counting and sizing in an optical microscope $(40 \times$ positive phase contrast objective). Previous experience had shown (9) that this procedure did not change the fiber length distribution.

\section{Evaluation of fibers in the eye}

The mucous thread is invisible to the naked eye but becomes easily visible after the instillation of alcian blue (7). The mucous thread can therefore be removed with two toothpicks and placed on a glass slide (5). A clump of blue mucus was clearly visible in the inner corner of the eyes of our subjects, and it could also be removed with a toothpick and placed on the same slide. In some cases there was also dried mucus (sleepy seeds) on the skin, and this material was removed as well. The slide was then left for drying.

Fibers could be seen in the dried thread and clump, although with difficulty, using an optical microscope $(40 \times$ positive phase contrast objective). The samples were therefore ashed in a low-temperature asher, and a coverslip was mounted. Measurement of the position of the fibers before and after ashing showed that this procedure did not change the position of the fibers. Great care was taken to keep air movements at an absolute minimum dur- 
ing the ashing procedure, especially during air admission. The nonrespirable fibers could easily be distinguished from the remaining structure of the mucus after ashing (fig 1, upper left). The fiber density was very high in some threads and clumps (fig 1, upper right), and problems occurred with counting and sizing the fibers. The possible error was minimized by the strict application of the following rule: Scan the sample and count and size those fibers with their endpoint entering the field of view. Scan areas exactly adjacent to each other.

The mucus was sampled from one eye only, since the other eye was examined with routine ophthalmologic methods for determining possible effects of the fibers. These results will be reported separately (Stokholm \& Schneider, in preparation).

\section{Results}

Only nonrespirable fibers were counted. The results are summarized in tables 1
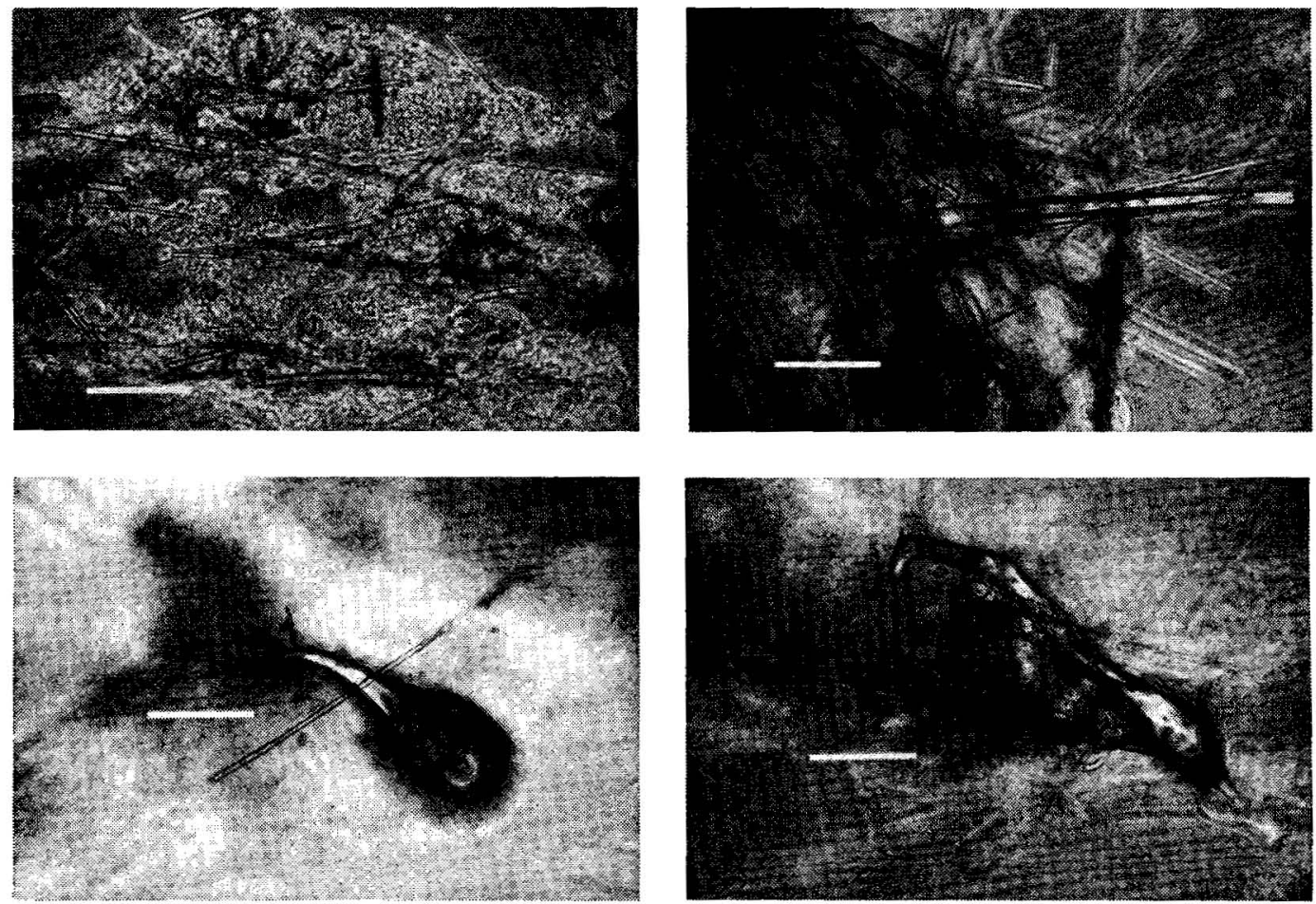

Fig 1. Photomicrographs of fibers in ashed mucus. - Upper left: accumulation in clump; upper right: high density of fibers in clump; lower left and right: peculiar shapes of fibers. (length of bar $0.1 \mathrm{~mm}$ ) 
Table 1. Total dust concentration and concentration of nonrespirable fibers in the total dust sample - Geometric mean and range.

Number of samples Total dust $\left(\mathrm{mg} / \mathrm{m}^{3}\right)$ Nonrespirable fibers $/ \mathrm{cm}^{3}$

Production work

Chamber work, material A

Chamber work, material B

$\begin{array}{llll}0.8 & (0.4-2.7) & 0.020 & (0.009-0.075) \\ 3.3 & (2.2-6.1) & 0.14 & (0.07-0.43) \\ 1.3 & (0.95-1.6) & 0.065 & (0.05-0.11)\end{array}$

Table 2. Number of nonrespirable fibers in the mucous thread and clump, including sleepy seeds - Geometric mean and range.

\begin{tabular}{lcc}
\hline & Number of samples & \multicolumn{2}{c}{ Nonrespirable fibers in thread + clump } \\
\cline { 2 - 4 } Production work & 10 & $55(9-250)$ \\
Chamber work & 5 & $30(10-60)$ \\
\hline
\end{tabular}

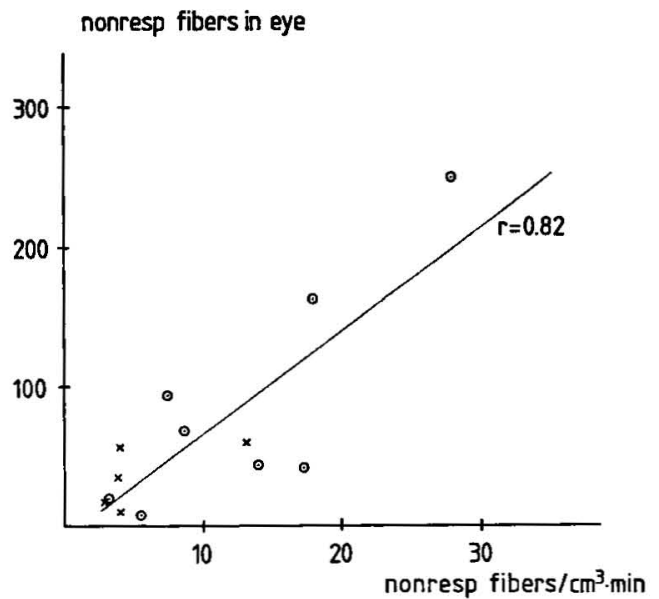

Fig 2. Total number of nonrespirable (nonresp) fibers accumulated in one eye vs airborne fiber exposure dose - Linear regression line and correlation coefficient. (o production work, $x$ chamber work)

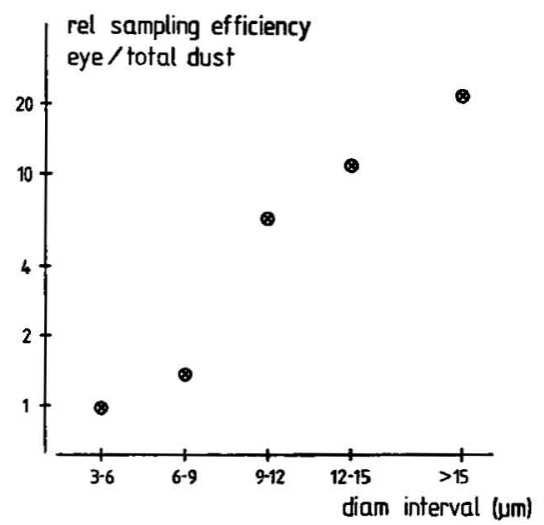

Fig 4. Diameter-dependent sampling efficiency of eye (fibers in thread + clump) relative (rel) to efficiency of total dust sampler. The points are calculated as number of fibers in eye/number of fibers in total dust sample and normalized to the interval 3-6 um. (diam: diameter)

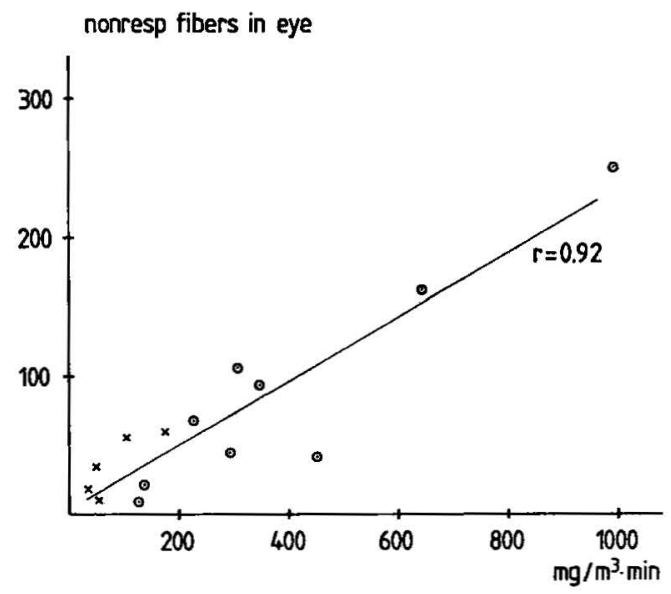

Fig 3. Total number of nonrespirable (nonresp) fibers accumulated in one eye vs total dust exposure dose - Linear regression line and correlation coefficient. (o production work, $\mathrm{x}$ chamber work)

\section{Discussion}

The better correlation seen in fig 3 as compared to that in fig 2 is explained in part by the better precision of weighing, as compared to the counting, of fibers on a filter.

Several factors will affect the correlation between the accumulated dust in the eyes and the dust exposure dose. Dust deposition will be dependent on the air currents across the face, and thus on the facial shape. Also, the exposed area of the eye varies.

It takes from 1 to $12 \mathrm{~h}$ for the clump in the inner corner of the eye to move onto the skin (1). There, it may be removed by 
rubbing with a finger. Although persons working with man-made mineral fibers usually avoid rubbing their eyes, there remains a risk of not having sampled all fibers which were deposited in the eye during the approximately $8 \mathrm{~h}$ of exposure during production work. Some oversampling may also have occurred in our investigation since the eye was not cleaned prior to the start of exposure.

Fig 2 and 3 could be described as the calibration curve for the human eye as a passive dust sampler. Alternatively, determining fibers in the mucus could be considered as biological monitoring with good correlation to ambient air sampling. Since fibers are deposited by sedimentation and impaction, a tendency for the eye to oversample thick fibers can be expected. This possibility is apparent from fig 4 . Fibers are deposited on the skin in a similar manner, and the present method could thus be used for assessing those work conditions which have a potential for causing skin irritation.

Further studies are needed to determine the sampling characteristics of the eye during a greater variety of exposure situations.

The lack of correlation between the number of fibers in the mucous thread and the fiber concentration during approximately the last half hour prior to the removal of the thread could be expected. On the basis of the study of 103 normal eyes, Norn (6) found an average migration rate of $1.1 \mathrm{~mm} / \mathrm{min}$, the single values ranging from nearly 0 to $2.5 \mathrm{~mm} / \mathrm{min}$. The migration rate is raised by frequent and intense blinking and reduced with decreased tear production. Thus, unless the migration rate of the mucous thread is known during the actual dust exposure, very little can be deduced with regard to the rate of deposition of dust into the eyes from studying the dust content in the thread. It is thus essential to sample all mucus, including sleepy seeds.

Because of surface tension, the fibers will be parallel with the surface of the eye after deposition. It could be expected that single, straight fibers only present minimal, if any, mechanical irritation to the eye. The situation may be different for fibers of peculiar shape (fig 1, lower left
\& right). The situation changes as the fibers migrate towards the inner corner of the eye. Here the mucous thread starts to curl up, the fibers form an intermingled structure, and fibers with lengths exceeding $1 \mathrm{~mm}$ point in all directions (fig 1 , upper right). It could be expected that these fibers present a mechanical irritation upon each blinking of the eye. A possibility exists that fibers lining up in the mucous thread can reduce the migration rate so that high fiber numbers could build up in the mucous thread.

\section{Conclusions}

The mucous thread in the lower fornix and the mucous clump in the corner of the eye become easily visible after the instillation of alcian blue and can be removed with toothpicks and placed on a slide. The sample is ashed in a low-temperature asher, a cover slip is placed on top, and the nonrespirable fibers are counted with the use of optical phase microscopy. This procedure requires a minimum of sample handling, and none of the fibers are displaced during the ashing procedure. It is essential to remove both the mucous thread in the lower fornix and the mucous clump in the inner corner of the eye, as well as the dry mucus on the skin, if present. The inner corner of the eye and the surrounding skin should be cleaned prior to the start of exposure.

The number of fibers in the mucous thread alone in a group of persons does not correlate with the dust concentration, whereas the number of accumulated nonrespirable fibers in the eye correlates well with the exposure dose based on the nonrespirable fibers in the total dust samples. The eye can thus be considered a passive dust sampler. Considered as such, the tendency to oversample thick fibers increases with increasing fiber diameter, when compared to the sampling efficiency of the total dust sampler with a small inlet opening pointing forward and having an inlet velocity of $1.25 \mathrm{~m} / \mathrm{s}$.

The eye is a target organ for industrial air pollutants, and the described method 
can be used as a biological monitoring method for exposure to man-made mineral fibers. It is suggested that this type of monitoring could be used to assess work conditions with potential for causing skin and eye irritation.

\section{Acknowledgment}

We wish to thank ED Smith and P Nissen, Rockwool International, for their cooperation and $\mathrm{J}$ Christiansen, Rockwool $\mathrm{A} / \mathrm{S}$, for having made all the practical arrangements in connection with the measurements. We also thank all volunteers (who let us remove the mucus without blinking too much) and $\mathrm{T}$ Rosenkrands for having done the fiber counting. We thank especially Prof MS Norn for his valuable suggestions.

Received for publication: 9 February 1981

\section{References}

1. Adams AD. The morphology of human conjunctival mucus. Arch ophthalmol 97 (1979) $730-734$

2. Gibson H, Vincent JH. On the impaction of airborne dust into the eyes of human subjects. Ann occup hyg 23 (1980) 35-45.

3. Harris RL, Fraser DA. A model for deposition of fibres in the human respiratory system. Am ind hyg assoc j 37 (1976) 73-89.

4. Lucas JB. The cutaneous and ocular effects resulting from worker exposure to fibrous dust. In: Proceedings of the symposium on the occupational exposure to fibrous glass, Maryland, June 26-27, 1974. US Department of Health Education and Welfare, Washington, DC 1976. (publication no (NIOSH) 76-151).

5. Norn MS. Mucous thread in inferior conjunctival fornix. Acta ophthalmol 44 (1966) $33-42$.

6. Norn MS. Mucous flow in the conjunctiva. Acta ophthalmol 46 (1968) 1125-1135.

7. Norn MS. External eye: Methods of examination. Scriptor, Copenhagen 1974.

8. Possick PA, Gellin GA, Key MM. Fibrous glass dermatitis. Am ind hyg assoc $\mathrm{j} 31$ (1970) 12-15.

9. Schneider T. Exposures to man-made mineral fibres in user industries in Scandinavia. Ann occup hyg 22 (1979) 153-162. 